\title{
Diagnostic en laboratoire de la maladie de Lyme
}

\author{
Lindsay LR $^{1^{*}}$, Bernat $K^{1}$ et Dibernardo $A^{1}$ \\ Laboratoire national de microbiologie, Agence de la santé publique du Canada, Winnipeg (Manitoba) \\ Auteur-ressource : robbin.lindsay@phac-aspc.gc.ca
}

\section{Résumé}

Contexte : La maladie de Lyme est en expansion au Canada. C'est une maladie à déclaration obligatoire. Au stade disséminé de l'infection, les épreuves sérologiques apportent des preuves complémentaires à l'appui de la confirmation des cas.

Objectif : Décrire les tests actuellement utilisés pour le diagnostic de la maladie de Lyme, passer en revue la démarche d'analyse recommandée pour les laboratoires et cerner les priorités futures de la recherche dans le domaine du diagnostic en laboratoire de la maladie de Lyme au Canada.

Méthodes: Une recherche documentaire a été effectuée. Nous avons ensuite résumé les paramètres à prendre en compte avant d'effectuer les tests de dépistage de la maladie de Lyme, décrit la meilleure pratique actuelle qui consiste à utiliser un algorithme de diagnostic en deux temps pour la confirmation en laboratoire de la maladie au stade disséminé, et analysé les avantages et les inconvénients des tests complémentaires concernant la maladie.

Résultats : Le recours à des tests diagnostiques est indiqué chez les patients présentant des symptômes du stade disséminé de la maladie et des antécédents d'exposition aux tiques vecteurs. Afin de maximiser la sensibilité et la spécificité des épreuves, le recours à un protocole d'essai en deux temps est recommandé. II consiste à effectuer un premier test de dépistage par dosage immunoenzymatique (EIA) suivi d'un test de confirmation par transfert Western. Un certain nombre d'autres tests diagnostiques sont disponibles; toutefois, ils sont surtout destinés à des fins de recherche.

Conclusion : L'analyse sérologique en deux temps est actuellement la meilleure démarche disponible pour faciliter la tâche des médecins lors de la pose d'un diagnostic de maladie de Lyme au stade disséminé.

L'Agence de la santé publique du Canada (l'Agence) s'efforcera d'améliorer cette démarche par la normalisation des tests diagnostiques de la maladie de Lyme sur l'ensemble des laboratoires du pays, par l'évaluation des caractéristiques de performance des tests des plateformes de diagnostic actuelles et futures et par l'établissement d'un processus destiné à établir des panels sériques fiables afin d'aider à la mise au point et à l'évaluation de nouveaux tests diagnostiques pour la maladie de Lyme.

\section{Introduction}

La maladie de Lyme est une infection transmise par des tiques et causée principalement par trois espèces de spirochètes du genre Borrelia burgdorferi sensu lato : $B$. burgdorferi sensu stricto (en Amérique du Nord et en Europe de l'Ouest), B. afzelii (en Europe de l'Ouest, en Europe centrale et en Russie) et B. garinii (principalement en Europe, en Russie et dans le nord de l'Asie)(1). Les symptômes de la maladie de Lyme se manifestent en plusieurs stades et concernent divers tissus et organes, notamment la peau, les articulations, le cœur et le système nerveux(2). L'incidence de la maladie de Lyme a augmenté de façon constante dans certaines parties du centre et de l'est du Canada (3-5) en raison de l'expansion récente du territoire de la principale tique vecteur, Ixodes scapularis(6).

La maladie de Lyme est une maladie à déclaration obligatoire au Canada depuis 2009(7). L'objectif de cet article est de décrire les tests diagnostiques en vigueur pour la maladie de Lyme, notamment de passer en revue les démarches recommandées pour l'analyse en laboratoire, ainsi que de cerner les priorités futures de la recherche sur le diagnostic de cette maladie au Canada. 


\section{Méthodes}

Une recension approfondie des publications à comité de lecture a été effectuée. Nous avons ensuite résumé les paramètres essentiels à prendre en compte avant d'effectuer les tests de dépistage de la maladie de Lyme, décrit la meilleure pratique actuelle qui consiste à utiliser un algorithme sérologique en deux temps pour la confirmation en laboratoire de la maladie au stade disséminé et exploré les avantages et les inconvénients des tests complémentaires concernant la maladie. Nous avons également donné un aperçu des futurs projets de recherche que le Laboratoire national de microbiologie de l'Agence prévoit d'entreprendre.

\section{Résultats}

\section{Facteurs à considérer avant d'effectuer les tests}

Au stade localisé précoce de la maladie de Lyme, il n'est pas nécessaire d'effectuer des tests diagnostiques avant d'instituer une antibiothérapie. Un diagnostic de présomption peut être posé en fonction du tableau clinique et d'antécédents crédibles d'exposition à des tiques à pattes noires infectées(8). En général, la réalisation de tests diagnostiques est appropriée chez les patients ayant des antécédents d'exposition à des tiques et présentant les symptômes d'une infection disséminée, puisque la sensibilité des tests augmente lorsque la bactérie touche des systèmes tissulaires autres que la peau $(8,9)$. Les tests doivent toutefois être réservés aux patients présentant des signes d'infection objectifs $(10,11)$.

Avant d'effectuer les tests, il est nécessaire d'obtenir les renseignements ci-dessous :

- Antécédents de voyage détaillés et date d'apparition des symptômes - Ces renseignements doivent être indiqués sur la demande d'analyse de laboratoire, puisqu'ils permettent au laboratoire de diagnostic d'utiliser la plateforme de test la plus appropriée. Par exemple, il existe des types de tests différents pour détecter la maladie de Lyme acquise en Europe ou en Asie et celle acquise en Amérique du Nord(12). De même, des tests différents sont utilisés pour les infections précoces et les infections susceptibles d'être plus anciennes(13).

- Antécédents d'antibiothérapie - Ils peuvent freiner la réponse immunitaire à l'infection et compliquer l'interprétation des résultats des tests sérologiques(14).

- Autres infections ou affections préexistantes - L'infection par d'autres agents pathogènes connexes (p. ex. syphilis) et l'existence de troubles auto-immuns peuvent entraîner l'obtention de résultats faussement positifs(15).

- Antécédents de cas confirmés en laboratoire de la maladie de Lyme - Ceci est important, car aucun schéma de réponse sérologique ne permet de distinguer une réinfection d'une infection initiale par B. burgdorferi(16).

\section{Dépistage de la maladie de Lyme}

Bien qu'il existe plusieurs stratégies de dépistage pouvant faciliter la pose du diagnostic de maladie de Lyme (tableau 1), la sérologie est actuellement la seule technique d'analyse de laboratoire normalisée disponible. Les paragraphes qui suivent décrivent les différentes plateformes de tests utilisées pour le diagnostic en laboratoire de la maladie de Lyme. Les avantages et les limites de chaque plateforme sont présentés au tableau 2. 
Tableau 1 :Stratégies de dépistage en laboratoire de la maladie de Lyme(9)

\begin{tabular}{|c|c|c|}
\hline Stade d'infection & $\begin{array}{l}\text { Stratégie de dépistage } \\
\text { recommandée* }\end{array}$ & Type d'échantillon \\
\hline $\begin{array}{l}\text { Érythème migrant, phase aiguë } \\
\text { (saison des tiques et exposition connue en } \\
\text { région d'endémie }{ }^{* *} \text { ) }\end{array}$ & Diagnostic clinique et traitement empirique & Aucun \\
\hline $\begin{array}{l}\text { Érythème migrant, phase aiguë } \\
\text { (hors saison ou aucune exposition connue en } \\
\text { région d'endémie) }\end{array}$ & $\begin{array}{l}\text { Sérologie en deux temps }{ }^{\dagger} \text {, répéter le dosage } \\
\text { EIA après quatre semaines en cas de } \\
\text { résultat négatif; traitement à la discrétion du } \\
\text { médecin; TAAN, isolement }\end{array}$ & $\begin{array}{l}\text { Sérique } \\
\text { Biopsie, plasma }\end{array}$ \\
\hline $\begin{array}{l}\text { Atteintes neurologiques, cardiaques ou } \\
\text { articulaires caractéristiques }\end{array}$ & $\begin{array}{l}\text { Sérologie en deux temps }{ }^{\dagger} \\
\text { TAAN }\end{array}$ & $\begin{array}{l}\text { Sérique } \\
\text { Liquide synovial ou } \\
\text { céphalorachidien }\end{array}$ \\
\hline $\begin{array}{l}\text { Symptômes persistants après l'administration du } \\
\text { traitement recommandé }\end{array}$ & Aucune & Aucune \\
\hline
\end{tabular}

\footnotetext{
* L'analyse sérologique est la technique de diagnostic la plus couramment utilisée; l'utilisation d'autres tests, notamment le test d'amplification des acides nucléiques (TAAN) ou l'isolement bactérien, est rare.

**On entend par régions endémiques, les régions où les tiques à pattes noires sont établies et où les cycles de transmission de B. burgdorferi sont maintenus.

+ Épreuve immunoenzymatique (EIA), suivie, le cas échéant, d'un test de confirmation par transfert Western à l'aide de trousses de diagnostic homologuées au Canada.
}

\section{Analyse sérologique}

Le Réseau des laboratoires de santé publique du Canada recommande l'utilisation d'une démarche en deux temps pour le diagnostic de la maladie de Lyme, soit une épreuve immunoenzymatique d'une sensibilité élevée (EIA) suivie, en présence d'un résultat positif ou indéterminé, d'un test par transfert Western d'une spécificité élevée(9). La raison du choix de cette démarche est que l'exécution en série de ces deux types de tests maximise la sensibilité et la spécificité d'ensemble de la sérologie.

La réponse immunitaire à l'infection par $B$. burgdorferi se traduit en premier lieu par l'apparition d'anticorps lgM, généralement dans les deux semaines suivant la morsure d'une tique(17). Ces anticorps peuvent persister pendant des mois, voire des années, même après un traitement antimicrobien efficace(18). Après l'apparition des IgM, des anticorps IgG sont exprimés chez la plupart des patients, habituellement un mois après la contraction de l'infection(9).

La sérologie fournit un aperçu du statut immunitaire du patient tel qu'il est au moment du prélèvement de l'échantillon. Par exemple, si la présence d'une maladie de Lyme est soupçonnée au vu des symptômes, mais que les tests sérologiques initiaux sont négatifs, la réalisation de tests de suivi sur un échantillon convalescent est recommandée(9). Les deux tests sérologiques les plus couramment effectués sont décrits ci-dessous.

\section{Épreuve immunoenzymatique (EIA)}

L'épreuve immunoenzymatique est utilisée à titre de test de dépistage pour détecter la présence dans le sérum d'anticorps IgM et IgG dirigés contre la bactérie responsable de la maladie de Lyme. Les trousses commerciales, notamment le test ELISA, reposent sur l'utilisation de préparations de cellules entières de $B$. burgdorferi(1) ou d'antigènes recombinants(19) (p. ex. peptide C6). La méthode d'essai autorise le dosage simultané d'un nombre relativement important d'échantillons. Bien que la plupart des tests immunoenzymatiques soient extrêmement sensibles, ils peuvent manquer de spécificité (p. ex. obtention de résultats faussement positifs due à la présence d'autres affections médicales).

\section{Test par transfert Western}

Le test par transfert Western est utilisé comme test de confirmation. Sa spécificité est supérieure à celle de l'épreuve immunoenzymatique $(11,20)$. II détecte la présence dans le sérum d'anticorps dirigés contre des extraits antigéniques séparés par électrophorèse et des antigènes recombinants de $B$. burgdorferi(21). Les trousses commerciales sont utilisées pour détecter la présence d'anticorps dirigés contre les génoespèces individuelles de Borrelia(12) et pour différencier les anticorps IgM des anticorps IgG. Un résultat positif au test 
par transfert Western est nécessaire pour confirmer l'exposition à $B$. burgdorferi(22); la séroconversion avec présence d'IgM puis d'IgG permet de confirmer l'existence d'une infection récente (9).

Tableau 2 : Tests de laboratoire pour la maladie de Lyme, leurs avantages et leurs limites

\begin{tabular}{ll}
\hline Test & Avantages \\
\hline Épreuve & - Permet de traiter de nombreux \\
immunoenzymatique & échantillons et est relativement facile \\
& à effectuer. \\
& - Fournit des valeurs numériques \\
& objectives contrairement à d'autres \\
& mesures subjectives (p. ex. dosages \\
& par immunofluorescence).
\end{tabular}

\section{Limites}

- Sensibilité moins élevée au stade précoce de la maladie de Lyme.

- Variabilité de sensibilité et de spécificité des diverses trousses commerciales disponibles au Canada.

- La présence d'anticorps dans le sérum de patients atteints de troubles auto-immuns, d'une infection par le virus Epstein-Barr, d'une endocardite bactérienne, d'une syphilis, d'autres spirochétoses, d'une anaplasmose ou d'une infection à Helicobacter pylori peut entraîner l'obtention de résultats faussement positifs.

- Certains tests ne peuvent pas faire la distinction entre les anticorps produits contre les génoespèces de Borrelia d'Amérique du Nord de ceux dirigés contre les génoespèces d'Europe et d'Asie.

- La sensibilité du test au stade précoce de la maladie peut varier en fonction du génotype de $B$. burgdorferi.

- Ne permet pas de différencier une infection à $B$. burgdorferi antérieure d'une réinfection.

Transfert Western

- Spécificité élevée permettant d'utiliser ce test pour écarter la responsabilité d'autres agents étiologiques.

- Permet de déterminer les classes d'immunoglobulines réactives (IgG ou $\lg M$ ) et aide à faire la distinction entre une infection au stade précoce et une infection plus ancienne.
- L'interprétation des résultats du test par transfert Western est subjective (disposition et intensité des bandes colorées) en l'absence de lecteur automatisé.

- Réaction croisée importante entre les diverses génoespèces européennes.

- Les anticorps IgM présentent une réactivité croisée inhérente qui peut entraîner l'obtention de résultats faussement positifs.

- Peut donner des résultats faussement négatifs pour les anticorps IgM au stade précoce de l'infection.

\begin{tabular}{|c|c|c|}
\hline \multicolumn{3}{|c|}{ TESTS DE LABORATOIRE COMPLÉMENTAIRES } \\
\hline Isolement bactérien & $\begin{array}{l}\text { - Spécificité élevée; pourrait être utile } \\
\text { pour déterminer les génotypes } \\
\text { de B. burgdorferi responsables de } \\
\text { l'infection. }\end{array}$ & $\begin{array}{l}\text { La collecte d'échantillons peut être invasive. } \\
\text { Test d'une sensibilité relativement faible, coûteux, } \\
\text { exigeant en main-d'œuvre; une longue période } \\
\text { d'incubation est nécessaire pour obtenir des résultats. }\end{array}$ \\
\hline $\begin{array}{l}\text { Test d'amplification } \\
\text { des acides } \\
\text { nucléiques (TAAN) }\end{array}$ & $\begin{array}{l}\text { - Permet de détecter l'ADN de } B \text {. } \\
\text { burgdorferi après l'institution d'une } \\
\text { antibiothérapie, et donc de } \\
\text { différencier une infection en cours } \\
\text { des symptômes persistants dus à un } \\
\text { mécanisme immunologique. } \\
\text { - L'ADN des borrélies peut être détecté } \\
\text { dans les érythèmes migrants avant } \\
\text { l'apparition des anticorps sériques et } \\
\text { plus rapidement que ne le permet } \\
\text { l'isolement bactérien. }\end{array}$ & $\begin{array}{l}\text { - Sensibilité peu élevée due à la faible charge } \\
\text { bactérienne de certains échantillons cliniques. } \\
\text { - Manque de normalisation en ce qui concerne les } \\
\text { gènes cibles. }\end{array}$ \\
\hline
\end{tabular}

\section{Algorithme en deux temps pour le diagnostic en laboratoire de la maladie de Lyme} La démarche en deux temps est illustrée à la figure 1. Dans un premier temps, on effectue une épreuve immunoenzymatique. Si les résultats sont négatifs, il n'y a pas lieu d'effectuer de test par transfert Western. Si 
les symptômes persistent, le test immunoenzymatique peut être répété sur un échantillon convalescent prélevé 3 à 6 semaines plus tard. Si les résultats de ce test sont positifs ou indéterminés, on passe au deuxième temps pour effectuer le test de confirmation par transfert Western. Au stade précoce de l'infection (symptômes présents depuis moins de six semaines), on effectue le test par transfert Western pour détecter à la fois les lgM et les IgG; si les symptômes sont présents depuis plus de six semaines, toutefois, seule la recherche des lgG par transfert Western est effectuée.

Figure 1. Analyse sérologique en deux temps pour le dépistage de la maladie de Lyme

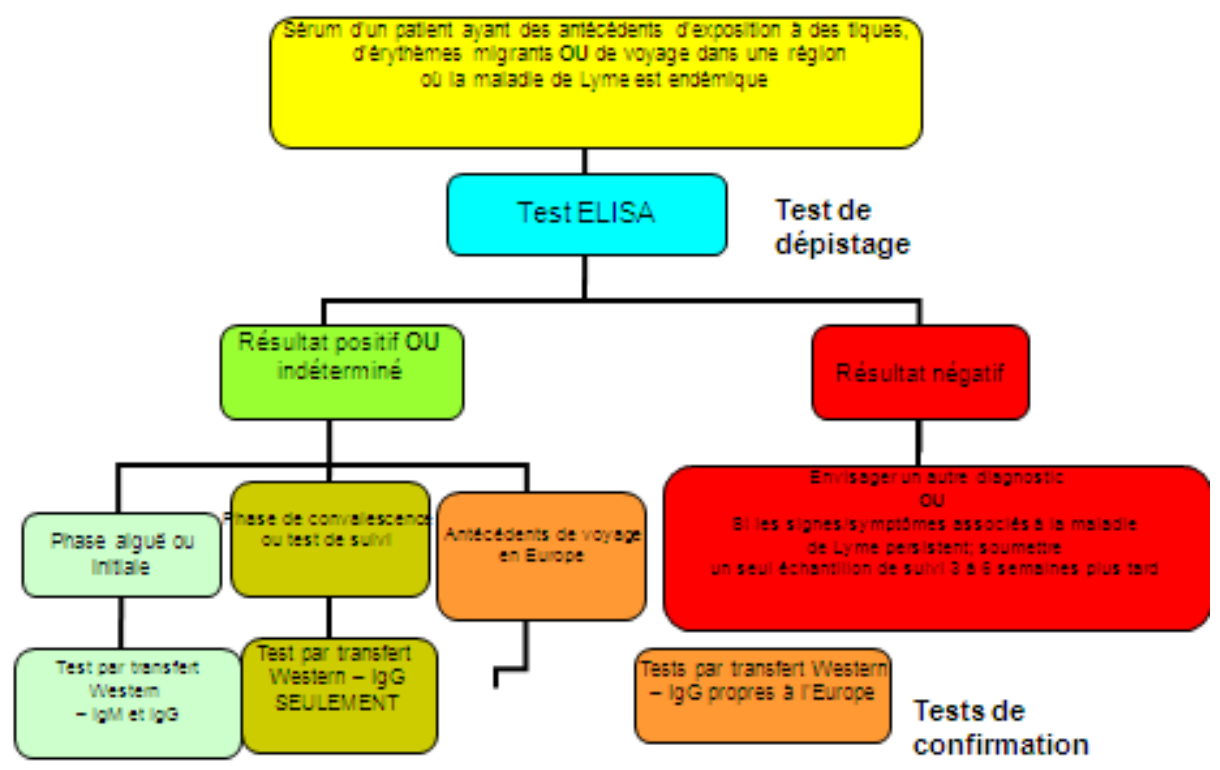

Le résultat final des tests sérologiques est considéré comme étant positif seulement lorsque le résultat de l'épreuve immunoenzymatique (EIA) est réactif (positif ou indéterminé) et que le test par transfert Western est également positif (tableau 3). La démarche en deux temps maximise la sensibilité et la spécificité des tests et augmente la probabilité de constater la séroconversion (IgM puis IgG) caractéristique des authentiques infections à $B$. burgdorferi( $1,17,21,23)$.

Tableau 3 : Interprétation des résultats du test par transfert Western (en conjonction avec des résultats EIA positifs ou indéterminés)

\begin{tabular}{ll}
\hline Résultat du test par transfert Western & Interprétation \\
\hline Test négatif pour lgM et IgG & $\begin{array}{l}\text { Ne correspond pas à une infection à B. burgdorferi; cependant, si les } \\
\text { symptômes persistent, soumettre un échantillon de suivi } 3 \text { à } 6 \\
\text { semaines plus tard. }\end{array}$ \\
Positif uniquement pour $\operatorname{lgM}^{*}$ & $\begin{array}{l}\text { Résultat potentiellement faussement positif s'il NE S'AGIT PAS d'un } \\
\text { cas aigu (apparition des symptômes datant de moins de } 6 \text { semaines). }\end{array}$ \\
Test positif uniquement pour lgG** & $\begin{array}{l}\text { Résultat conforme à une infection à } B \text {. burgdorferi contractée plus de } \\
4 \text { semaines avant le test. } \\
\text { Indique une infection à } B \text {. burgdorferi récente ou des antécédents } \\
\text { d'infection. }\end{array}$ \\
\hline
\end{tabular}


** Test positif pour IgM - présence de 2 bandes significatives sur 3.

Test positif pour lgG - présence de 5 bandes significatives sur 10(22).

IgM = immunoglobuline $M$.

IgG = immunoglobuline $\mathrm{G}$.

\section{Autres tests de laboratoire de détection de B. burgdorferi}

\section{Isolement bactérien}

L'isolement de $B$. burgdorferi viable dans des échantillons cliniques nécessite l'incubation de l'échantillon dans un milieu de culture spécialisé. Bien que ce test demeure la référence pour le diagnostic de la maladie de Lyme (7), la méthode est coûteuse, dénuée de sensibilité clinique et comporte des risques de contamination (24). Sur le plan pratique, elle est surtout limitée par la durée d'incubation des cultures qui peut atteindre jusqu'à huit semaines en raison du petit nombre d'organismes viables présent dans de nombreux types de l'échantillon (17). Pour ces raisons, ses possibilités d'application clinique sont limitées et son emploi réservé aux études de recherche (9).

\section{Test d'amplification des acides nucléiques (TAAN)}

Le test d'amplification des acides nucléiques (TAAN) est utilisé pour accélérer l'obtention des résultats des tests diagnostiques de la maladie de Lyme $(25,26)$. Plusieurs techniques de réaction en chaîne de la polymérase (PCR) (c.-à-d. PCR imbriquée, en temps réel ou quantitative) sont utilisées pour amplifier diverses cibles génétiques spécifiques à Borrelia dans les échantillons cliniques (27). Les résultats positifs sont plus souvent observés au stade précoce de la maladie (28). La sensibilité de la réaction en chaîne de la polymérase sur le liquide céphalorachidien (LCR) est faible ou variable et, par conséquent, d'une utilité limitée dans le cadre de l'évaluation des patients atteints de signes neurologiques $(1,21)$. Bien que ce test permette une détection plus précoce de l'infection que les tests sérologiques $(25,27)$, à l'heure actuelle, son utilisation est limitée aux études de recherche $(8,9)$.

\section{Défis associés aux tests diagnostiques de la maladie de Lyme}

Les médecins et les chercheurs de laboratoire jugent préoccupante la variabilité qui existe entre les résultats obtenus par certains laboratoires privés et ceux obtenus par les laboratoires de santé publique du Canada. II semblerait qu'un certain nombre de laboratoires privés utilisent des tests ou des critères d'interprétation qui n'ont pas été suffisamment validés. L'utilisation de tests insuffisamment validés dont l'exactitude n'a pas été adéquatement établie peut entraîner la production de résultats faussement positifs.

Parmi ces tests, citons le dosage par immunocapture des antigènes dans l'urine, la coloration par immunofluorescence, le tri cellulaire des formes kystiques et à paroi cellulaire déficiente de $B$. burgdorferi, les tests de transformation des lymphocytes (29) et une nouvelle méthode de culture de sérum (30).

À l'heure actuelle, le non-respect de l'algorithme en deux temps (p. ex. transfert Western effectué seul ou après un test EIA négatif) peut augmenter la fréquence d'obtention de résultats faussement positifs. Ce qui peut en retour entraîner des erreurs de diagnostic et l'administration inutile de traitements (1). Il est impératif que les cliniciens soient conscients des idées fausses qui courent sur la maladie de Lyme (31) et connaissent les meilleures pratiques de laboratoire destinées à son diagnostic (1); cela leur permettrait de répondre de façon éclairée aux questions et préoccupations soulevées par leurs patients.

\section{Évolution future}

Comme il a été mentionné, il y existe une grande diversité de tests diagnostiques pour aider à diagnostiquer la maladie de Lyme (21,32), l'exactitude et la fiabilité de certains de ces tests faisant l'objet d'une forte controverse. (32-34). À I'heure actuelle, aucun test diagnostique n'offre $100 \%$ de sensibilité et de spécificité pour la confirmation de la maladie de Lyme. La grande variabilité de l'expression clinique de l'infection à $B$. burgdorferi chez les patients complique davantage encore la situation. L'amélioration des plateformes de tests diagnostiques est une priorité. La recherche de " biomarqueurs » de la maladie de Lyme indépendants de la réponse sérologique est en cours (35). Des variantes de la démarche en deux temps, visant généralement à limiter ou éliminer le recours au test par transfert Western, sont en cours d'évaluation (36-39). Elles pourraient simplifier l'interprétation des tests et améliorer leur sensibilité au stade précoce de la maladie. 
L'un des plus grands défis auxquels l'évaluation de nouvelles démarches diagnostiques est confrontée (40) au Canada est le manque de panels sériques ou de pools d'échantillons parfaitement caractérisés provenant de cas confirmés d'infection à $B$. burgdorferi. II est également impératif de bien connaître les génotypes de $B$. burgdorferi responsables des infections contractées par les Canadiens (41) et de déterminer la capacité des tests diagnostiques actuellement utilisés à les détecter tous avec une sensibilité comparable.

Le Laboratoire national de microbiologie de l'Agence envisage de travailler de concert avec les laboratoires de diagnostic à l'échelle du Canada afin d'examiner les pratiques de diagnostic de la maladie de Lyme et les systèmes d'assurance de la qualité actuellement en place ainsi que d'évaluer la nécessité d'améliorer les tests internes et externes de vérification des compétences. À long terme, l'Agence prévoit également les actions suivantes : 1) examiner et mettre à jour les lignes directrices actuelles des laboratoires relatives à la maladie de Lyme; 2) déterminer et comparer les caractéristiques de performance de toutes les plateformes utilisées au Canada pour les épreuves immunoenzymatiques et les tests par transfert Western; 3) accorder une attention prioritaire et continue à l'évaluation de nouvelles plateformes de diagnostic; et 4) amorcer le processus de mise au point d'un panel sérique fiable destiné à l'évaluation de nouveaux tests (42). Ces actions permettront d'améliorer la qualité des tests diagnostiques pour la maladie de Lyme au Canada.

\section{Conclusion}

L'incidence de la maladie de Lyme est à la hausse au Canada. En présence d'un érythème migrant et d'antécédents fiables d'exposition à des tiques à pattes noires, le dépistage n'est pas nécessairement requis et le traitement peut être institué de façon prospective. L'évaluation clinique des patients au stade disséminé de la maladie de Lyme peut être étayée par des analyses de laboratoire.

À l'heure actuelle, il n'existe pas de test de laboratoire parfait pour la maladie de Lyme; la démarche sérologique en deux temps offre à ce jour les taux de sensibilité et de spécificité les plus élevés. Une utilisation incorrecte des tests sérologiques ou l'utilisation de tests diagnostiques ou de critères d'interprétation insuffisamment validés peuvent aboutir à un diagnostic erroné et à une antibiothérapie inutile. Les efforts futurs seront axés sur la normalisation des tests et sur la mise au point et l'évaluation de nouvelles démarches diagnostiques dans le but d'optimiser la détection de la maladie de Lyme.

\section{Remerciements}

Nous tenons à remercier nos collègues des laboratoires de diagnostic provinciaux et des laboratoires d'hôpitaux de partout au Canada pour les commentaires importants et utiles dont ils nous ont fait part sur la démarche de diagnostic en laboratoire de la maladie de Lyme du Laboratoire national de microbiologie.

\section{Conflit d'intérêts}

Les auteurs n'ont aucun conflit d'intérêts à déclarer. 


\section{Références}

(1) Johnson BJB, Aguero-Rosenfeld ME, Wilske B. Sood SK, editor. Lyme borreliosis in Europe and North America: epidemiology and clinical practice. [Internet]. Hoboken, NJ: John Wiley \& Sons, Inc.; 2011. Serodiagnosis of Lyme borreliosis; p. 185-212. [consulté le 28 novembre 2013]. DOI: 10.1002/9780470933961.ch10

(2) Hatchette TF, Davis I, Johnston BL. Lyme disease: clinical diagnosis and treatment. CCDR. 2014; 40-11:215231.

(3) Ogden NH, Lindsay LR, Morshed M, Sockett PN, Artsob H. The emergence of Lyme disease in Canada. CMAJ [Internet]. 2009 [consulté le 28 novembre 2013]; 180(12):1221-4.

(4) Ogden NH, Artsob H, Lindsay LR, Sockett PN. Lyme disease: A zoonotic disease of increasing importance to Canadians. Can Fam Phys [Internet]. 2008; 54(10):1381-4.

(5) Ogden, N.H., Lindsay, L.R., Morshed, M., Sockett, P.N., Artsob, H. La borréliose de Lyme au Canada : un problème grandissant. Relevé des maladies transmissibles au Canada (Internet), vol. 34(1), p. 1-19, 2008.

(6) Ogden, N.H., Koffi, J.K., Pelcat, Y., Lindsay, L.R. Risque environnemental pour la maladie de Lyme dans l'est et le centre du Canada : un sommaire d'informations récentes en matière de surveillance. Relevé des maladies transmissibles au Canada, vol. 40(5), p. 74-82, 2014.

(7) Définitions nosologiques des maladies transmissibles faisant l'objet d'une surveillance nationale - 2009. Relevé des maladies transmissibles au Canada [Internet], vol. 35(supplément 2), p. 1-123, novembre 2009.

(8) Wormser GP, Dattwyler RJ, Shapiro ED, Halperin JJ, Steere AC, Klempner MS, Krause PJ, Bakken JS, Strle F, Stanek G, Bockenstedt L, Fish D, Dumler JS, Nadelman RB. The clinical assessments treatment, and prevention of Lyme disease, human granulocytic anaplasmosis, and babesiosis: Clinical practice guidelines by the Infectious Diseases Society of America. Clin Infect Dis [Internet]. 2006; 43(9):1089-134.

(9) Canadian Public Health Laboratory Network. The laboratory diagnosis of Lyme borreliosis: Guidelines from the Canadian Public Health Laboratory Network. Can J Infect Dis Med Microbiol [Internet]. Mar 2007; 18(2):145-8.

(10) Seltzer EG, Shapiro ED. Misdiagnosis of Lyme disease: When not to order serologic tests. Pediatr Infect Dis J [Internet]. 1996; 15(9):762-3.

(11) Tugwell P, Dennis DT, Weinstein A, Wells G, Shea B, Nichol G, Hayward R, Lightfoot R, Baker P, Steere AC. Laboratory evaluation in the diagnosis of Lyme disease. Ann Intern Med [Internet]. Dec 15 1997; 127(12):1109-23.

(12) Branda JA, Strle F, Strle K, Sikand N, Ferraro MJ, Steere AC. Performance of United States serologic assays in the diagnosis of Lyme borreliosis acquired in Europe. Clin Infect Dis [Internet]. Aug 2013; 57(3):333-40.

(13) Schoen RT. Better laboratory testing for Lyme disease: No more Western blot. Clin Infect Dis [Internet]. 2013 [consulté le 27 novembre 2013]; 57(3):341-3.

(14) Aguero-Rosenfeld ME, Nowakowski J, Bittker S, Cooper D, Nadelman RB, Wormser GP. Evolution of the serologic response to Borrelia burgdorferi in treated patients with culture-confirmed erythema migrans. J Clin Microbiol [Internet]. 1996; 34(1):1-9.

(15) Johnson BJ, Robbins KE, Bailey RE, Cao BL, Sviat SL, Craven RB, Mayer LW, Dennis DT. Serodiagnosis of Lyme disease: accuracy of a two-step approach using a flagella-based ELISA and immunoblotting. J Infect Dis [Internet]. Aug 1996; 174(2):346-53.

(16) Nadelman RB, Wormser GP. Reinfection in patients with Lyme disease. Clin Infect Dis [Internet]. Oct 15 2007; 45(8):1032-8. 
(17) Aguero-Rosenfeld ME, Wang G, Schwartz I, Wormser GP. Diagnosis of Lyme borreliosis. Clin Microbiol Rev [Internet]. Jul 2005; 18(3):484-509.

(18) Kalish RA, McHugh G, Granquist J, Shea B, Ruthazer R, Steere AC. Persistence of immunoglobulin M or immunoglobulin $\mathrm{G}$ antibody responses to Borrelia burgdorferi 10-20 years after active Lyme disease. Clin Infect Dis [Internet]. 2001; 33(6):780-5.

(19) Liang FT, Alvarez AL, Gu Y, Nowling JM, Ramamoorthy R, Philipp MT. An immunodominant conserved region within the variable domain of VlsE, the variable surface antigen of Borrelia burgdorferi. J Immunol [Internet]. 1999; 163(10):5566-73.

(20) Dressler F, Whalen JA, Reinhardt BN, Steere AC. Western blotting in the serodiagnosis of Lyme disease. J Infect Dis [Internet]. Feb 1993; 167(2):392-400.

(21) Aguero-Rosenfeld, ME. Lyme disease: laboratory issues. Infect Dis Clin North Am [Internet]. 2008 [consulté le 27 novembre 2013]; 22(2):301-13.

(22) Centers for Disease Control and Prevention (CDC). Recommendations for test performance and interpretation from the Second National Conference on Serologic Diagnosis of Lyme Disease. MMWR Morb Mortal Wkly Rep [Internet]. Aug 11 1995; 44(31):590-1.

(23) Craven RB, Quan TJ, Bailey RE, Dattwyler R, Ryan RW, Sigal LH, Steere AC, Sullivan B, Johnson BJ, Dennis DT, Gubler DJ. Improved serodiagnostic testing for Lyme disease: results of a multicenter serologic evaluation. Emerg Infect Dis [Internet]. Apr-Jun 1996; 2(2):136-40.

(24) Nowakowski J, Schwartz I, Liveris D, Wang G, Aguero-Rosenfeld ME, Girao G, McKenna D, Nadelman RB, Cavaliere LF, Wormser GP, Lyme Disease Study Group. Laboratory diagnostic techniques for patients with early Lyme disease associated with erythema migrans: a comparison of different techniques. Clin Infect Dis [Internet]. Dec 15 2001; 33(12):2023-7.

(25) Wormser GP, Wang G, Sood SK, editor. Lyme borreliosis in Europe and North America : epidemiology and clinical practice. [Internet]. Hoboken, N.J.: John Wiley \& Sons, Inc.; 2011The role of culture and nucleic acid amplification in diagnosis of Lyme borreliosis; p. 159-83. DOI: 10.1002/9780470933961.ch9.

(26) Dumler JS. Molecular diagnosis of Lyme disease: review and meta-analysis. Mol Diagn [Internet]. Mar 2001; 6(1):1-11.

(27) Eshoo MW, Schutzer SE, Crowder CD, Carolan HE, Ecker DJ. Achieving molecular diagnostics for Lyme disease. Expert Rev Mol Diagn [Internet]. Nov 2013; 13(8):875-83.

(28) Liveris D, Schwartz I, McKenna D, Nowakowski J, Nadelman R, Demarco J, Iyer R, Bittker S, Cooper D, Holmgren D, Wormser GP. Comparison of five diagnostic modalities for direct detection of Borrelia burgdorferi in patients with early Lyme disease. Diagn Microbiol Infect Dis [Internet]. Jul 2012; 73(3):243-5.

(29) Centers for Disease Control and Prevention (CDC). Caution regarding testing for Lyme disease. Morb Mortal Wkly Rep [Internet]. 2005; 54(5):125.

(30) Johnson BJ, Pilgard MA, Russell TM. Assessment of New Culture Method to Detect Borrelia species in Serum of Lyme Disease Patients. J Clin Microbiol [Internet]. Aug 142013.

(31) Halperin JJ, Baker P, Wormser GP. Common misconceptions about Lyme disease. Am J Med [Internet]. 2013 [consulté le 28 novembre 2013]; 126(3):264.e1,264.e7.

(32) Johnson BJB. Halperin JJ, editor. Lyme Disease: An Evidence-based Approach. Cambridge, MA, USA: CABI Publishing, 2011. Laboratory diagnostic testing for Borrelia burgdorferi infection; p. 73-88.

(33) Bowie WR. Guidelines for the management of Lyme disease: The controversy and the quandary. Drugs [Internet]. 2007; 67(18):2661-6. 
(34) Halperin JJ, Baker P, Wormser GP. Halperin JJ, editor. Lyme disease: An Evidence-based Approach. Cambridge, MA, USA: CABI Publishing, 2011. Lyme Disease: the Great Controversy; p. 259-70.

(35) Cerar T, Ogrinc K, Lotric-Furlan S, Kobal J, Levicnik-Stezinar S, Strle F, Ruzic-Sabljic E. Diagnostic value of cytokines and chemokines in Lyme neuroborreliosis. Clin Vaccine Immunol [Internet]. Oct 2013; 20(10):1578-84.

(36) Porwancher RB, Hagerty CG, Fan J, Landsberg L, Johnson BJB, Kopnitsky M, Steere AC, Kulas K, Wong SJ. Multiplex immunoassay for Lyme disease using VIsE1-IgG and pepC10-IgM antibodies: Improving test performance through bioinformatics. Clin Vaccine Immunol [Internet]. 2011; 18(5):851-9.

(37) Wormser GP, Schriefer M, Aguero-Rosenfeld ME, Levin A, Steere AC, Nadelman RB, Nowakowski J, Marques A, Johnson BJB, Dumler JS. Single-tier testing with the C6 peptide ELISA kit compared with two-tier testing for Lyme disease. Diagn Microbiol Infect Dis [Internet]. 2013; 75(1):9-15.

(38) Branda JA, Linskey K, Kim YA, Steere AC, Ferraro MJ. Two-tiered antibody testing for Lyme disease with use of 2 enzyme immunoassays, a whole-cell sonicate enzyme immunoassay followed by a vlse c6 peptide enzyme immunoassay. Clin Infect Dis [Internet]. 2011; 53(6):541-7.

(39) Branda JA, Aguero-Rosenfeld ME, Ferraro MJ, Johnson BJB, Wormser GP, Steere AC. 2-Tiered antibody testing for early and late Lyme disease using only an immunoglobulin $\mathrm{G}$ blot with the addition of a VlsE band as the second-tier test. Clin Infect Dis [Internet]. 2010; 50(1):20-6.

(40) Ogden NH, Lindsay LR, Morshed M, Sockett PN, Artsob H. The emergence of Lyme disease in Canada. CMAJ [Internet]. 2009 [consulté le 28 novembre 2013]; 180(12):1221-4.

(41) Ogden NH, Margos G, Aanensen DM, Drebot MA, Feil EJ, Hanincová K, Schwartz I, Tyler S, Lindsay LR. Investigation of genotypes of Borrelia burgdorferi in Ixodes scapularis ticks collected during surveillance in Canada. Appl Environ Microbiol [Internet]. 2011; 77(10):3244-54.

(42) Aguero-Rosenfeld ME. Committee on Lyme Disease and Other Tick-Borne Diseases: The State of the Science, Institute of Medicine., editors. Critical needs and gaps in understanding prevention, amelioration, and resolution of Lyme and other tick-borne diseases [electronic resource] : the short-term and long-term outcomes : workshop report. [Internet]. Washington, DC: National Academies Press; 2011. Diagnostics for Lyme disease: Knowledge gaps and needs; p. 125-30. 